INPLASY

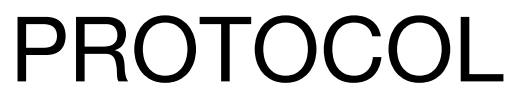

To cite: Liu et al.

Consciousness-

restoring and orifice-

opening acupuncture for delayed recovery A protocol

for systematic review and

network meta-analysis. Inplasy

protocol 2021120110. doi:

10.37766/inplasy2021.12.0110

Received: 24 December 2021

Published: 24 December 2021

Corresponding author:

Ningning Liu

Inn0927@163.com

Author Affiliation:

Affiliated Hospital of

Shandong University of

Traditional Chinese

Medicine.

Support: Shandong

University of TCM.

Review Stage at time of this submission: Preliminary

searches.

Conflicts of interest:

None declared.

\section{Consciousness-restoring and orifice- opening acupuncture for delayed recovery A protocol for systematic review and network meta-analysis}

\author{
Liu, N1; Xu, Y2; Zhang, D3; Wang, L4; Hou, Y5; Ji, J6.
}

Review question / Objective: How do Consciousnessrestoring and orifice-opening acupuncture treat delayed recovery?

Condition being studied: A systematic evaluation of the effect of Consciousness-restoring and orifice-opening acupuncture on delayed recovery.

Information sources: We will search foreign and Chinese databases, including PubMed, EMBASE, MEDLINE, CENTRAL, CNKI, WanFang Data, CBM, and VIP from the inception of the coverage of these databases to December 2021. Data, CBM, and VIP from the inception of the coverage of these databases to December 2020. The databases will be retrieved by combining the subject words with random words. Taking PubMed as an example, the retrieval strategy is shown in Table 1. The search terms will be adapted appropriately to conform to the different syntax rules of the different databases.

INPLASY registration number: This protocol was registered with the International Platform of Registered Systematic Review and Meta-Analysis Protocols (INPLASY) on 24 December 2021 and was last updated on 24 December 2021 (registration number INPLASY2021120110).

\section{INTRODUCTION}

Review question / Objective: How do Consciousness-restoring and orifice- opening acupuncture treat delayed recovery?

Condition being studied: A systematic evaluation of the effect of Consciousness- 
restoring and orifice-opening acupuncture on delayed recovery.

\section{METHODS}

Participant or population: Patients with delayed recovery.

Intervention: The intervention measures in the experimental group should contain Consciousness-restoring and orificeopening acupuncture.

Comparator: As included in eligible randomized clinical trials and retrospective cohort studies.

Study designs to be included: Randomized clinical trials and retrospective cohort studies will be included.

Eligibility criteria: Participants were patients with delayed recovery after laparoscopic cholecystitis resection, regardless of age, gender, ethnicity. At present, it is believed that when general anesthesia is stopped as planned, if the patient cannot recover consciousness within 60 minutes and cannot make thinking answers or movements to speech or stimuli, etc., it can be considered as delayed recovery.

Information sources: We will search foreign and Chinese databases,including PubMed, EMBASE, MEDLINE, CENTRAL, CNKI, WanFang Data, CBM, and VIP from the inception of the coverage of these databases to December 2021. Data, CBM, and VIP from the inception of the coverage of these databases to December 2020. The databases will be retrieved by combining the subject words with random words. Taking PubMed as an example, the retrieval strategy is shown in Table 1. The search terms will be adapted appropriately to conform to the different syntax rules of the different databases.

Main outcome(s): The effective rate of clinical symptoms, the scores of each index (recovery time, respiration, skin color, consciousness, blood pressure, finger pulse oxygen saturation, heart rate).
Quality assessment / Risk of bias analysis: Two researchers (XL and $\mathrm{YH}$ ) will assess the quality of the included RCTs independently by utilizing the Cochrane Risk of Bias assessment tool. As specified by the Cochrane Handbook (5.2.0), the following sources of bias will be considered: random sequence generation, allocation concealment, participant blinding, outcome assessor blinding, incomplete outcome data, selective reporting, and other sources of bias. Each domain will be rated as having a high, low, or unclear risk of bias as appropriate.[13] The 2 reviewers will resolve any disagreements through discussion, and a third reviewer (TD) will be consulted if no consensus is reached.

Strategy of data synthesis: The RevMan5.3 software provided by the Cochrane website will be used for the analysis. Categorical variables will be expressed by odds ratio (OR) and marked with $95 \%$ confidence interval $(\mathrm{Cl})$. Continuous variables will be expressed by mean difference (MD) and marked with $95 \%$ Cl. If $\mathbf{P 0 . 1}$ or $\left.\right|^{2}<50 \%$, it means that the heterogeneity between groups is small, and the fixed effect model will, in this case, be used for combined analysis; when $\mathrm{P} 50 \%$, it shows that the heterogeneity between the groups is large, and the random effects model will then be used for combined analysis, and the results shown in forest plots.Analysis of potential publication bias will be shown in a funnel chart, and sensitivity analysis and subgroup analysis will be used when necessary.

Subgroup analysis: Subgroup analysis will be used to evaluate the therapeutic effects among different drugs.Inverted funnel plots and Egger's regression test will be used to determine publication bias when the number of included studies exceeds 10 in the network meta-analysis.

Sensitivity analysis: If the heterogeneity is high, we will also perform subgroup analysis to calculate the combined statistics. The following subgroup analyses will be considered: sex, age, intervention 
time, intervention cycle, and duration of intervention.

Country(ies) involved: China.

Keywords: consciousness-restoring and orifice-opening acupuncture, delayed recovery, laparoscopic cholecystectomy, general anesthesia, network meta-analysis, protocol, systematic review, traditional Chinese medicine.

Contributions of each author:

Author 1 - Ningning Liu.

Author 2 - Yingxue Xu.

Author 3 - Dongbin Zhang.

Author 4 - Lianzhu Wang.

Author 5 - Yi Hou.

Author 6 - Jiafu Ji. 